Regards sur l'économie allemande

Bulletin économique du CIRAC

$86 \mid 2008$

Varia

\title{
Audiovisuel : l'avenir protégé du service public allemand
}

Isabelle Bourgeois

\section{OpenEdition}

Édition électronique

URL : http://journals.openedition.org/rea/906

DOI : $10.4000 /$ rea. 906

ISBN : 978-2-8218-0868-3

ISSN : 1965-0787

\section{Éditeur}

CIRAC

Édition imprimée

Date de publication : 1 mai 2008

Pagination : 37-38

ISSN : 1156-8992

\section{Référence électronique}

Isabelle Bourgeois, «Audiovisuel : I'avenir protégé du service public allemand », Regards sur l'économie allemande [En ligne], 86 | mai 2008, mis en ligne le 01 mai 2010, consulté le 15 septembre 2020. URL : http://journals.openedition.org/rea/906 


\section{Actualité économique}

\section{AUDIOVISUEL : 1'avenir protégé du service public allemand}

L'annonce du projet de suppression de la publicité sur les chaînes publiques françaises a apporté de l'eau au moulin des télévisions privées allemandes qui voient d'un mauvais œil proliférer l'offre concurrente du service public le plus richement doté d'Europe. Cela fait longtemps qu'elles rêvent d'une mesure analogue outre-Rhin pour mettre fin à une concurrence qu'elles jugent faussée par ce financement mixte... Mais leur rêve est loin de devenir réalité. Non seulement, ARD et ZDF devraient conserver 'leur' publicité encore longtemps, mais elles verront aussi augmenter 'leur' redevance au $1^{\mathrm{er}}$ janvier 2009.

Le marché audiovisuel allemand, le plus dynamique d'Europe, se distingue du français sur de nombreux points. Sur l'état de la concurrence dans la télévision, déjà : l'offre standard gratuite est d'une trentaine de chaînes, moitié publiques, moitié privées ; leur réception s'effectue essentiellement par câble et satellite, considérés comme de simples vecteurs de distribution (le hertzien terrestre ne concerne plus que quelque 1,9 millions de foyers sur un total de 37 millions). L'offre complémentaire à péage (un peu plus de 3,5 millions d'abonnés) n'est jamais réellement parvenue à s'imposer face à cette offre gratuite pléthorique. Dans le segment gratuit, l'audience se répartit à peu près à égalité entre public et privé ; au sein du pôle privé, les deux principaux opérateurs (RTL Group et ProSiebenSat.1) se partagent eux aussi l'audience à égalité (voir REA 75/06). En un mot: aucune grande chaîne ne voit sa part d'audience dépasser significativement le seuil des 12 \%. Sur le marché de la publicité TV (20,3 milliards $€$ en 2006$)$, avec environ $8 \%$, la part du service public peut être considérée comme résiduelle ; le reste se partage pratiquement à égalité entre les deux pôles privés. Ces grands volumes sont stables depuis une décennie.

Le basculement au numérique, en cours, n'a pas le même impact qu'en France, où la TNT est synonyme d'une démultiplication de l'offre. En Allemagne, la numérisation passe pour l'instant surtout par la réception satellite, et l'offre, largement semblable à celle du mode analogique, devrait rester majoritairement gratuite. La réception numérique par satellite est en outre favorisée par la forte concurrence existant sur le marché des récepteurs où les prix d'entrée de gamme se situent à $30 €$. Ce faible prix, rendant accessible la TV numérique à plus de 8 millions de foyers, est rendu possible, contrairement à la France, par l'absence de cryptage du signal (au moyen de technologies propriétaires) des chaînes analogiques gratuites dupliquées en numérique sur le satellite Astra. La diffusion terrestre est aussi résiduelle en numérique qu'en analogique. En revanche, le mode filaire (le plus souvent le câble, qui dessert plus de 18 millions de foyers en analogique) pourrait se développer rapidement, compte tenu des 3 millions de foyers actuellement câblés en numérique, et de la progression fulgurante de l'équipement informatique des ménages; le seul handicap est le prix encore souvent dissuasif des démodulateurs. Ce n'est donc pas tant, contrairement à la situation française, via le numérique terrestre (et encore, la TNT restera un phénomène largement urbain de ce côté-ci du Rhin) que l'offre globale va se diversifier en Allemagne, mais via le câble et le satellite, de même que le réseau téléphonique.

Opérateurs publics et privés de l'audiovisuel se développent donc en conséquence, élargissant leur offre aussi bien en matière de télévision que de contenus en ligne, convergence oblige. Or c'est là que le bât blesse aux yeux des opérateurs privés. Le service public investit massivement ces 'nouveaux' médias, en concurrence frontale avec les opérateurs privés, mais aussi les éditeurs de presse. Durant l'exercice quadriennal 2005-08, elles auront consacré 33,5 millions $€$ au développement de contenus en ligne $(0,5$ million pour l'établissement Deutschlandradio) - soit 0,75\% seulement de leurs dépenses. II est vrai que, en comparaison française, elles ne manquent pas de moyens.

Budget 2005-08 des établissements de radiodiffusion de droit public en RFA (en millions $€$ )

\begin{tabular}{|c|c|c|c|c|}
\hline & ARD & ZDF & DRadio & Total \\
\hline Total recettes, dont: & 24183 & 7954 & 801 & 32938 \\
\hline -redevance & 20752 & 6874 & 725 & 28201 \\
\hline -publicité a) & 388 & 452 & - & 823 \\
\hline -autres ${ }^{\text {b) }}$ & 3043 & 628 & 77 & 3748 \\
\hline
\end{tabular}
Source des données : 16. KEF-Bericht, décembre 2007. NB : Calculs effectués par l'auteur ; chiffres arrondis. a) dont sponsoring,
cession de droits ou produit de placements financiers; $b$ ) les radios de DeutschlandRadio ne diffusent pas de publicité ; elle est autorisée sur les chaînes de l'ARD et de la ZDF seulement les jours ouvrables entre 17 et 20 heures.

Avec $17,03 €$ par mois depuis 2005 , la redevance est non seulement deux fois plus élevée en Allemagne qu'en France (son produit s'élevait à plus de 7 milliards $€$ en 2006), mais devrait augmenter encore de 95 cents en janvier prochain. C'est en tout cas le montant préconisé pour la période 2009-2012 par la Commission chargée de l'évaluation des besoins

\section{Analyse}

Chaînes publiques : la moitié de l'audience et $8 \%$ de la publicité

La dynamique du numérique portée par la convergence

La TV se développe en ligne

La redevance passera à 17,98 € par mois en 2009 
Redevance : large assiette, faible taux d'exonération

Un statut certes particulier outre-Rhin...

... mais un prélèvement public aux yeux de la Commission

Pour le justifier, il faut définir les missions du service public

La Constitution ne le permet guère

Un conflit cornélien pour la politique audiovisuelle financiers des établissements de radiodiffusion de droit public (Kommission zur Ermittlung des Finanzbedarfs der Rundfunkanstalten, KEF) dans son dernier rapport, présenté le 21 janvier 2008. Encore faut-il que les ministres-présidents des Länder confèrent force de loi à cette augmentation en modifiant ad hoc leur Contrat d'Etat sur la redevance.

Or statut et structure de la redevance allemande constituent un second élément de distinction avec la situation française. La redevance est prélevée, comme naguère encore en France, par récepteur et non par foyer. Certes, un certain nombre de ménages sont exonérés pour diverses raisons, mais le taux d'exonération reste très bas (8\% seulement). Ensuite, la base de la redevance est plus large: elle est prélevée mensuellement sur les récepteurs radio (actuellement: $5,52 €$ ) et les téléviseurs $(11,51 €)$; depuis le $1^{\text {er }}$ janvier 2007, le même taux que pour la radio est également prélevé sur les « récepteurs audiovisuels nouveaux », c'est-à-dire les ordinateurs et autres équipements électroniques comme les portables (81 000 récepteurs déclarés au $1^{\text {er }}$ juillet 2007).

Enfin, en droit, la redevance n'est pas une taxe ; il s'agit au contraire d'une sorte de cotisation due par la collectivité pour ce service d'intérêt général dont le prestataire n'est pas l'Etat (ce qu'interdirait la Constitution), mais la collectivité des citoyens qui délègue cette mission publique à des établissements autonomes et indépendants, dotés du statut de collectivités de droit public, ce qui implique leur souveraineté en matière d'administration et de gestion : les établissements de radiodiffusion de droit public (Rundfunkanstalten). Le contrôle de leur gestion est effectué a posteriori par la Cour des comptes de chaque Land. En effet, ces établissements ne sont pas nationaux, puisque leur création relève du législateur du Land (souverain en matière de culture). Les Länder peuvent conclure des traités pour fonder un établissement commun, ce qui est le cas de la ZDF, établissement de tous les Länder, ou du RBB, commun à Berlin et au Brandebourg. Si chaque établissement est indépendant pour la ligne éditoriale développée (la Constitution interdit les cahiers des charges, assimilée à une ingérence de l'Etat), la conformité de son action avec la mission d'intérêt général qui lui est confiée est contrôlée par la collectivité. Ce contrôle est donc exercé par une sorte de conseil de surveillance composé de représentants de la société civile organisée (églises, syndicats, patronat, associations de parents d'élèves...).

Or si ce statut spécifique s'accompagne d'un financement particulier, celui-ci n'en constitue pas moins un prélèvement public, d'autant que son montant est fixé en derniers recours (par voir contractuelle) par les ministres-présidents des Länder, autrement dit l'exécutif (c'est là une incohérence du droit allemand des médias, par ailleurs très formaliste sur l'indépendance). Cette situation avait amené la Commission européenne à engager en 2005 une procédure pour aide publique d'Etat contre la République fédérale. Si l'affaire est close à ce niveau, les Länder s'étant engagés à réformer les points litigieux d'ici 2009 en adaptant la législation, elle est source de virulents débats en Allemagne.

Au cœur d'entre eux se trouve, comme dans tout secteur des services d'intérêt général en voie de libéralisation, le financement mixte des acteurs publics. Or ce sujet préoccupe actuellement tous les Etats membres de l'UE dans le cadre de la définition, à l'échelon communautaire, du cadre futur du financement étatique des services publics de radiodiffusion, dossier sur lequel la procédure de consultation s'est achevée le 10 mars 2008. Pour justifier un tel financement dans un environnement aujourd'hui libéralisé et largement concurrentiel, il s'agit entre autres de définir précisément la mission du service public de l'audiovisuel. Or c'est là que le bât blesse.

La Loi fondamentale, via la jurisprudence du Tribunal constitutionnel fédéral, définit cette mission comme par nature «au service » de la démocratie, ce rôle particulier requérant l'indépendance des acteurs qui, pour pouvoir l'exercer pleinement, doivent disposer de moyens budgétaires suffisants. Cet impératif de moyens inclut la publicité, complément de recettes bienvenu et régulateur indirect de l'adéquation entre la demande et l'offre de programmes. En droit, la publicité jouit de la même garantie constitutionnelle que l'information.

Le conflit est cornélien pour l'audiovisuel classique : définir plus précisément cette mission revient à la réduire, au mieux par la loi, mais même cela constituerait une violation de l'indépendance des médias. Réduire la part publique (redevance) du financement est indispensable à la fois pour inciter les établissements à une gestion plus rigoureuse des deniers publics et pour alléger le poids des prélèvements sur les ménages. Mais comment faire? La Cour de Karlsruhe a invalidé le 11-09-2007 le dernier traité conclu par les Länder et dans lequel ils faisaient injonction aux établissements de rationaliser leur fonctionnement et refusaient d'augmenter la redevance dans les proportions prônées par la KEF pour 2005-08; le législateur doit veiller à assurer un financement permettant au service public de remplir ses missions - y inclus le développement dans les nouveaux services. Et il doit surtout mieux justifier, le cas échéant, son désir de réduire les prélèvements au nom de l'intérêt général. Kurt Beck (SPD), ministre-président de Rhénanie-Palatinat et président de la Conférence permanente des Länder sur l'audiovisuel résumait ainsi le dilemme de la politique audiovisuelle allemande : «le grand écart entre Karlsruhe et Bruxelles ». (IB) 